\title{
Some Sequence of Wrapped $\Delta$-Labellings for the Complete Bipartite Graph
}

\author{
Tomoko Adachi, Daigo Kikuchi \\ Department of Information Sciences, Toho University, Funabashi, Japan \\ Email: adachi@is.sci.toho-u.ac.jp
}

Received 2 January 2015; accepted 17 January 2015; published 20 January 2015

Copyright (C) 2015 by authors and Scientific Research Publishing Inc.

This work is licensed under the Creative Commons Attribution International License (CC BY). http://creativecommons.org/licenses/by/4.0/

(c) (†) Open Access

\begin{abstract}
The design of large disk array architectures leads to interesting combinatorial problems. Minimizing the number of disk operations when writing to consecutive disks leads to the concept of "cluttered orderings" which were introduced for the complete graph by Cohen et al. (2001). Mueller $e t$ al. (2005) adapted the concept of wrapped $\Delta$-labellings to the complete bipartite case. In this paper, we give some sequence in order to generate wrapped $\Delta$-labellings as cluttered orderings for the complete bipartite graph. New sequence we give is different from the sequences Mueller $e t$ al. gave, though the same graphs in which these sequences are labeled.
\end{abstract}

\section{Keywords}

Cluttered Ordering, RAID, Disk Arrays, Label for a Graph

\section{Introduction}

The desire to speed up secondary storage systems has lead to the development of disk arrays which achieve performance through disk parallelism. While performance improves with increasing numbers of disks, the chance of data loss coming from catastrophic failures, such as head crashes and failures of the disk controller electronics, also increases. To avoid high rates of data loss in large disk arrays, one includes redundant information stored on additional disks_-also called check disks — which allows the reconstruction of the original datastored on the so-called information disks-even in the presence of disk failures. These disk array architectures are known as redundant arrays of independent disks (RAID) (see [1] [2]).

Optimal erasure-correcting codes using combinatorial framework in disk arrays are discussed in [1] [3]. For an optimal ordering, there are [4] [5]. Cohen et al. [6] gave a cyclic construction for a cluttered ordering of the complete graph. In the case of a complete graph, there are [7] [8]. Furthermore, in the case of a complete bipartite graph, Mueller et al. [9] gave a cyclic construction for a cluttered ordering of the complete bipartite graph by 
utilizing the notion of a wrapped $\Delta$-labelling. In the case of a complete tripartite graph, we refer to [10].

As Figure 1, we present the case $\ell=2$. For example, information disk 1 is associated to the check disks $a$ and c. A 2-dimensional parity code can be modeled by the complete bipartite graph $K_{\ell, \ell}=(U, V, E)$ in the following way. The point set of $K_{\ell, \ell}$ is partitioned into the two sets- $U$ and $V$ both having cardinality $\ell$. Assign the points of $U$ to the $\ell$ check bits corresponding to the rows and the points of $V$ to the $\ell$ check bits corresponding to the columns. By definition, in $K_{\ell, \ell}$ any point of $U$ is connected with any point of $V$ exactly on edge constituting the edge set $E$, i.e., $|E|=\ell^{2}$ (see Figure 2).

In this paper, we make label to the vertex of a bipartite graph. For example, we make label 1, 3, 0 and -1 , respectively, to four vertices $a, b, c$ and $d$ of a bipartite graph in Figure 2. By such labelling, we get that the label of the edge $\{a, c\}$ is $1-0=1$; the label of the edge $\{a, d\}$ is $1-(-1)=2$; the label of the edge $\{b, c\}$ is $3-0=3$ and the label of the edge $\{b, d\}$ is $3-(-1)=4$. The labellings $[1,3]$ of the upper vertices $[a, b]$ and the labellings $[0,-1]$ of the lower vertices $[c, d]$ are sequences. The goal of this paper is to find new sequence in order to generate wrapped $\Delta$-labellings as cluttered orderings for the complete bipartite graph. In Section 5, we give new sequence which we want. The new sequence we give is different from the sequences Mueller et al. [9] gave, though the same graphs in which these sequences are labeled.

\section{A Cluttered Ordering}

In a RAID system disk writes are expensive operations and should therefore be minimized. In many applications there are writes on a small fraction of consecutive disks-say $d$ disks-where $d$ is small in comparison to $k$, the number of information disks. Therefore, to minimize the number of operations when writing to $d$ consecutive information disks one has to minimize the number of check disks-say $f$-associated to the $d$ information disks.

Let $G=(V, E)$ be a graph with $n=|V|$ vertices and edge set $E=\left\{e_{0}, e_{1}, \cdots, e_{m-1}\right\}$. Let $d \leq m$ be a positive integer, called a window of $G$, and $\pi$ a permutation on $\{0,1, \cdots, m-1\}$, called an edge ordering of $G$. Then, given a graph $G$ with edge ordering $\pi$ and window $d$, we define $V_{i}^{\pi, d}$ to be the set of vertices which are connected by an edge of $\left\{e_{\pi(i)}, e_{\pi(i+1)}, \cdots, e_{\pi(i+d-1)}\right\}, 0 \leq i \leq m-1$, where indices are considered modulo $m$. The cost of accessing a subgraph of $d$ consecutive edges is measured by the number of its vertices. An upper bound of this cost is given by the $d$-maximum access cost of $G$ defined as $\max _{i}\left|V_{i}^{\pi, d}\right|$. An ordering $\pi$ is a ( $d$, f)-cluttered ordering, if it has $d$-maximum access cost equal to $f$. We are interested in minimizing the parameter $f$.

Let $\ell$ be a positive integer and let $K_{\ell, \ell}$ denote the complete bipartite graph with $2 \ell$ vertices and $\ell^{2}$ edges. In the following, we identify the vertex set of $K_{\ell, \ell}$ with $Z_{\ell} \times Z_{2}$, where two vertices are connected by an edge iff they have different second components in $Z_{\ell} \times Z_{2}$. The construction of $(d$, $f$ )-cluttered orderings for $K_{\ell, \ell}$ with small positive integer $f$ is based on two fundamental concepts. Firstly, we introduce the well-known concept of a $\Delta$-labelling of a suitable bipartite subgraph from which one gets a decomposition of $K_{\ell, \ell}$ into isomorphic copies of this subgraph. Secondly, we define the concept of a $(d$, f $)$-movement which will lead to "locally" defined edge orderings of $K_{\ell, \ell}$. This principle was implicitely used in [6] in case of the complete graph. In case of the complete bipartite graph, we refer to [9].

In the following, $H=(U, E)$ always denotes a bipartite graph with vertex set $U$ which is partitioned into

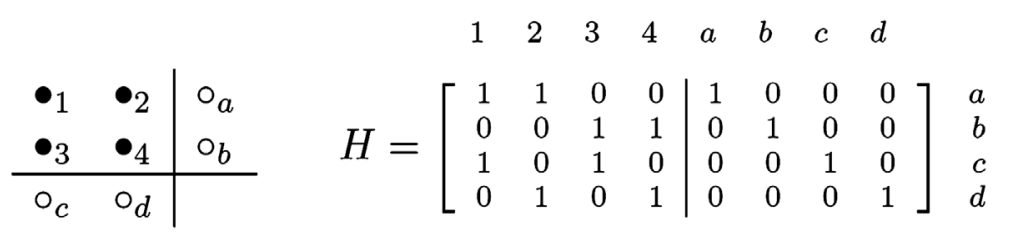

Figure 1. 2-dim. parity code and its parity check matrix.

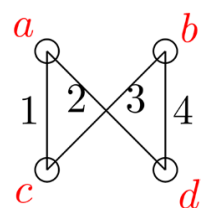

Figure 2. Code as graph. 
two subsets denoted by $V$ and $W$. Any edge of the edge set $E$ contains exactly one point of $V$ and $W$ respectively. Let $\ell=|E|$, then a $\Delta$-labelling of $H$ with respect to $V$ and $W$ is defined to be a map $\Delta: U \rightarrow Z_{\ell} \times Z_{2}$ with $\Delta(V) \subset Z_{\ell} \times\{0\}$ and $\Delta(W) \subset Z_{\ell} \times\{1\}$, where each element of $Z_{\ell}$ occurs exactly once in the difference list

$$
\Delta(E):=\left(\pi_{1}(\Delta(v)-\Delta(w)) \mid v \in V, w \in W,(v, w) \in E\right) .
$$

Here, $\pi_{1}: Z_{\ell} \times Z_{2} \rightarrow Z_{\ell}$ denotes the projection on the first component. In general, $\Delta$-labellings are a wellknown tool for the decomposition of graphs into subgraphs (see [11]). In this context a decomposition is understood to be a partition of the edge set of the graph. In case of the complete bipartite graph, one has the following proposition.

Proposition 1. ([9]) Let $H=(U, E)$ be a bipartite graph, $\ell=|E|$, and $\Delta$ be a $\Delta$-labelling as defined above. Then there is a decomposition of the complete bipartite graph $K_{\ell, \ell}$ into isomorphic copies of $H$.

For example, Figure 3 shows $\Delta$-labellings of a graph $H=H(1 ; 1)$ with 3 edges leading to a decomposition of $K_{3,3}$ into isomorphic copies of $H(1 ; 1)$ such as Figure 4. Next, in order to move a graph $H$ to an isomorphic copy such as Figure 5, we define the concept of a $(d, f)$-movement which can easily be generalized to arbitrary set system.

Definition 1. Let $G$ be a graph with edge set $E(G)=\left\{e_{0}, e_{1}, \cdots, e_{n-1}\right\}$, where $n$ is positive integer, and let $\Sigma_{0}$, $\Sigma_{1} \subset E(G)$ with $d:=\left|\Sigma_{0}\right|=\left|\Sigma_{1}\right|$. For a permutation $\sigma$ on $\{0,1, \cdots, n-1\}$ define $V_{i}^{\sigma, d}:=\bigcup_{j=0}^{d-1} e_{\sigma(i+j)}$ for $0 \leq i \leq n-d$. Then, for some given a positive integer $f$, and a map $\sigma$ is called a $(d, f)$-movement from $\Sigma_{0}$ to $\Sigma_{1}$ if $\Sigma_{0}=\left\{e_{\sigma(j)} \mid 0 \leq j \leq d-1\right\}, \quad \Sigma_{1}=\left\{e_{\sigma(j)} \mid n-d \leq j \leq n-1\right\}$, and $\max _{i}\left|V_{i}^{\sigma, d}\right| \leq f$.

In order to assemble such $(d, f)$-movements of certain subgraphs to a $(d, f)$-cluttered ordering, we need some notion of consistency. Let $\varphi: \Sigma_{0} \rightarrow \Sigma_{1}$ be any bijection, then a $\left(d\right.$, $f$ )-movement $\sigma$ from $\Sigma_{0}$ to $\Sigma_{1}$ is called consistent with $\varphi$ if

$$
\varphi\left(e_{\sigma(j)}\right)=e_{\sigma(n-d+j)}, \quad \text { for } \quad j=0,1, \cdots, d-1 .
$$

Now, for each $j \in Z_{\ell}$ one gets an automorphism $\tau_{j}$ of the bipartite graph $K_{\ell, \ell}$ defined by cyclic translation of the vertex set:
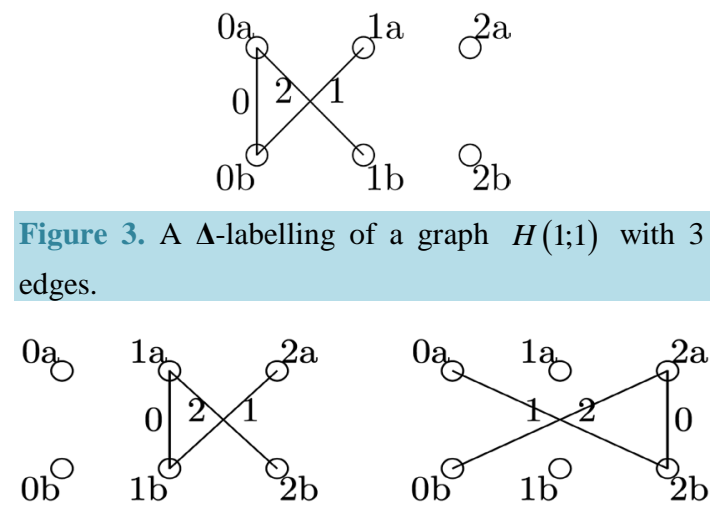

Figure 4. Isomorphic copies of $H(1 ; 1)$.
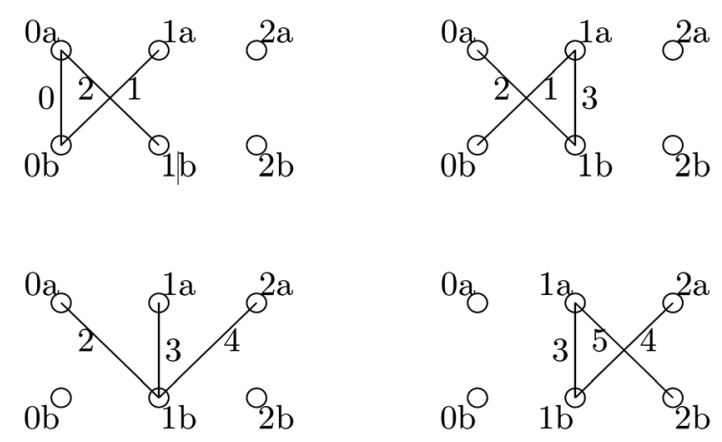

Figure 5. A (3,4)-movement. 


$$
\tau_{j}: Z_{\ell} \times Z_{2} \rightarrow Z_{\ell} \times Z_{2}, \quad \tau_{j}((u, b)):=(u+j, b),
$$

$(u, b) \in Z_{\ell} \times Z_{2}$. Obviously, $\tau_{j}$ induces in a natural way an automorphism of the edge set of $K_{\ell, \ell}$ which we also denote $\tau_{j}$. Then, $\tau_{j}\left(E^{(i)}\right)=E^{(i+j)}$ and $\tau_{j}\left(\Sigma_{0}^{(i)}\right)=\Sigma_{0}^{(i+j)}, i \in Z_{\ell}$. Next, we define a subgraph $G^{(0)} \subset K_{\ell, \ell}$ by specifying its edge set $E\left(G^{(0)}\right):=E^{(0)} \cup \Sigma_{0}^{(\kappa)}$. Let $E\left(G^{(0)}\right)=\left\{e_{0}^{(0)}, e_{1}^{(0)}, \cdots, e_{n-1}^{(0)}\right\}, n=\ell+d$, where we fix some arbitrary edge ordering. We denote the restriction of the cyclic translation $\tau_{\kappa}$ to $\Sigma_{0}^{(0)}$ by $\varphi_{\kappa}^{(0)}$ which defines a bijection $\varphi_{\kappa}^{(0)}: \Sigma_{0}^{(0)} \rightarrow \Sigma_{0}^{(\kappa)}$.

Definition 2. With above notation, a $\left(d\right.$, f)-movement of $G^{(0)}$ from $\Sigma_{0}^{(0)}$ to $\Sigma_{0}^{(\kappa)}$ consistent with $\varphi_{\kappa}^{(0)}$ will be denoted as $(d, f)$-movement from $\Sigma_{0}^{(0)}$ consistent with the translation parameter $\kappa$.

According to Definition 1, such a $(d, f)$-movement is given by some permutation $\sigma$ of the index set $\{0,1, \cdots, n-1\}$. By applying the cyclic translation $\tau_{i}$ one gets a graph $G^{(i)}:=\tau_{i}\left(G^{(0)}\right)$ with edge set $E\left(G^{(i)}\right)=E^{(i)} \cup \Sigma_{0}^{(i+\kappa)}=\left\{e_{0}^{(i)}, e_{1}^{(i)}, \cdots, e_{n-1}^{(i)}\right\}, \quad i \in Z_{\ell}$. We denote the restriction of $\tau_{\kappa}$ to $\Sigma_{0}^{(i)}$ by $\varphi_{\kappa}^{(i)}$ which defines a bijection

$$
\varphi_{\kappa}^{(i)}: \Sigma_{0}^{(i)} \rightarrow \Sigma_{0}^{(i+\kappa)}, \quad \varphi_{\kappa}^{(i)}\left(e^{(i)}\right)=e^{(i+\kappa)}, \quad e^{(i)} \in \Sigma_{0}^{(i)} .
$$

Then $\sigma$ also defines a $(d, f)$-movement of $G^{(i)}$ from $\Sigma_{0}^{(i)}$ to $\Sigma_{0}^{(i+\kappa)}$ consistent with $\varphi_{\kappa}^{(i)}$. Using that $e_{\sigma(j)}^{(i)} \in \Sigma_{0}^{(i)}, \quad 0 \leq j<d$, (see Defintion 1), we get, for $j=0,1, \cdots, d-1$,

$$
e_{\sigma(j)}^{(i+\kappa)} \stackrel{(4)}{=} \varphi_{\kappa}^{(i)}\left(e_{\sigma(j)}^{(i)} \stackrel{(2)}{=} e_{\sigma(n-d+j)}^{(i)}=e_{\sigma(\ell+j)}^{(i)} .\right.
$$

Having such a consistent $\sigma$, it is easy to construct a $(d$, f $)$-cluttered ordering of $K_{\ell, \ell}$. In short, one orders the edges of $K_{\ell, \ell}$ by first arranging the subgraphs of the decomposition along $E^{(0)}, E^{(\kappa)}, E^{(2 \kappa)}, \cdots, E^{((\ell-1) \kappa)}$ and then ordering the edges within each subgraph according to $\sigma$.

Proposition 2. ([9]) Let $H=(U, E), \quad \ell=|E|$, be a bipartite graph allowing some $\rho$-labelling, and let $\kappa$ be a translation parameter coprime to $\ell$. Furthermore, let $\Sigma_{0} \subset E, d:=\left|\Sigma_{0}\right|$. If there is a $(d, f)$-movement from $\Sigma_{0}$ consistent with $\kappa$, then there also is a $(d$, f)-cluttered ordering for the complete bipartite graph $K_{\ell, \ell}$.

\section{Construction of Cluttered Orderings of $H(h ; t)$}

In this section, we define an infinite family of bipartite graphs which allow $(d, f)$-movements with small $f$. In order to ensure that these $(d, f)$-movements are consistent with some translation parameter $\kappa$, we impose an additional condition on the $\Delta$-labellings also referred to as wrapped-condition.

Let $h$ and $t$ be two positive integers. For each parameter $f$ and $t$, we define a bipartite graph denoted by $H(h ; t)=(U, E)$. Its vertex set $U$ is partitioned into $U=V \cup W$ and consists of the following $2 h(t+1)$ vertices:

$$
\begin{aligned}
& V:=\left\{v_{i} \mid 0 \leq i<h(t+1)\right\}, \\
& W:=\left\{w_{i} \mid 0 \leq i<h(t+1)\right\} .
\end{aligned}
$$

The edge set $E$ is partitioned into subsets $E_{s}, 0 \leq s<t$, defined by

$$
\begin{aligned}
& E_{s}^{\prime}:=\left\{\left\{v_{i}, w_{j}\right\} \mid s \cdot h \leq i, j<s \cdot h+h\right\}, \\
& E_{s}^{\prime \prime}:=\left\{\left\{v_{i}, w_{h+j}\right\} \mid s \cdot h \leq j \leq i<s \cdot h+h\right\}, \\
& E_{s}^{\prime \prime \prime}:=\left\{\left\{v_{h+i}, w_{j}\right\} \mid s \cdot h \leq i \leq j<s \cdot h+h\right\}, \\
& E_{s}:=E_{s}^{\prime} \cup E_{s}^{\prime \prime} \cup E_{s}^{\prime \prime \prime}, \quad \text { for } \quad 0 \leq s<t, \\
& E:=\bigcup_{s=0}^{t-1} E_{s} .
\end{aligned}
$$


Figure 6 shows the edge partition of $H(2 ; 1)$. For the number of edges holds

$$
|E|=t \cdot\left(h^{2}+\frac{h(h+1)}{2}+\frac{h(h+1)}{2}\right)=t h(2 h+1) .
$$

The $t$ subgraphs defined by the edge sets $E_{s}, 0 \leq s<t$, and its respective underlying vertex sets are isomorphic to $H(h ; 1)$. Intuitively speaking, the bipartite graph $H(h ; t)$ consists of $t$ "consecutive" copies of $H(h ; 1)$, where the last $h$ vertices of $V$ and $W$ respectively of one copy are identified with the first $h$ vertices of $V$ and $W$ respectively of the next copy. Traversing these copies with increasing $s$ will define a $(d, f)$-movement of $H(h ; t)$ with small parameter $f$ as is shown in the next proposition.

Proposition 3. ([9]) Let $h, t$ be pogitive integers. Let $H(h ; t)=(U, E), t \geq 2$, be the bipartite graph as defined above. Then, there is a $(d, f)$-movement of $H(h ; t)$ from $E_{0}$ to $E_{t-1}$ with $d=h(2 h+1)$ and $f=4 h$.

By Proposition 1 a $\Delta$-labelling of the graph $H(h ; t)$ will lead to a decomposition of the complete bipartite graph $K_{\ell, \ell}$ into $\ell$ isomorphic copies of $H(h ; t)$, where $\ell=t h(2 h+1)$. However, in general there is no $(d, f)$-movement consistent with some translation parameter $\kappa$. To this means, we impose an additional condition on the $\Delta$-labelling. The following definition generalizes and adapts the notion of a wrapped $\Delta$-labelling to the bipartite case, which was introduced in [6] for certain subgraphs of the complete graph.

Definition 3. Let $H=(U, E), \ell=|E|$, denote a bipartite graph and let $X, Y \subset U$ with $|X|=|Y|$. A $\Delta$ labelling $\Delta$ is called a wrapped $\Delta$-labelling of $H$ relative to $X$ and $Y$ if there exists a $\kappa \in Z$ coprime to $\ell$ such that

$$
\Delta(Y)=\Delta(X)+(\kappa, 0)
$$

as multisets in $Z_{\ell} \times Z_{2}$. The parameter $\kappa$ is also referred to as translation parameter of the wrapped $\Delta$-labelling.

For the graphs $H=H(h ; t)$, we define $X:=\left\{v_{i}, w_{i} \mid 0 \leq i<h\right\}$ and $Y:=\left\{v_{i}, w_{i} \mid h t \leq i<h(t+1)\right\}$. Furthermore, in the following we only consider wrapped $\Delta$-labellings relative to $X$ and $Y$ for which the stronger condition

$$
\Delta\left(v_{i+h t}\right)=\Delta\left(v_{i}\right)+(\kappa, 0) \text { and } \Delta\left(w_{i+h t}\right)=\Delta\left(w_{i}\right)+(\kappa, 0),
$$

hold for $0 \leq i<h$. Suppose we have such labelling $\Delta$ satisfying condition (7). Now, $E^{(i)}, i \in Z_{\ell}$, are isomorphic copies of $H(h ; t)$. Furthermore, $\Sigma_{0}^{(\kappa)}$ is isomorphic to $H(h ; 1)$ consisting of the first $d$ edges of $E^{(\kappa)}$. From condition (7) follows that the graph $G^{(0)} \subset K_{\ell, \ell}$ with edge set $E\left(G^{(0)}\right):=E^{(0)} \cup \Sigma_{0}^{(\kappa)}$ can obviously identified with $H(h ; t+1)$. In addition, one easily checks that the $(d, f)$-movement of $G^{(0)}=H(h ; t+1)$ from Proposition 3 is consistent with the translation parameter $\kappa$.

Proposition 4. ([9]) Let $h, t$ be positive integers. From any wrapped $\Delta$-labelling of $H(h ; t)$, satisfying condition (7), one gets a $(d, f)$-cluttered ordering of the complete bipartite graph $K_{\ell, \ell}$ with $\ell=t h(2 h+1)$, $d=h(2 h+1)$, and $f=4 h$.

\section{Sequences of Wrapped $\Delta$-Labellings for $H(1 ; t), H(2 ; t)$ and $H(h ; 1)$}

In this section, we construct some infinite families of such wrapped $\Delta$-labellings. By applying Proposition 2 we get explicite $(d, f)$-cluttered orderings of the corresponding bipartite graphs. For these results in this section, we refer to [9].

\subsection{A Sequence for $H(1 ; t)$}

We define a wrapped $\Delta$-labelling of $H(1 ; t)$ for any positive integer t. $H(1 ; t)=(U, E)$ has $2(t+1)$ vertices

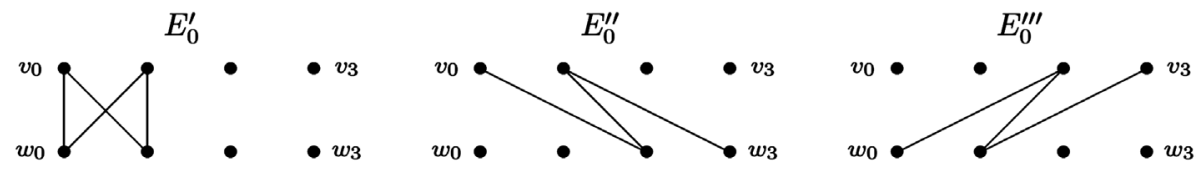

Figure 6. Partition of the edge set of $H(2 ; 1)$. 
and $3 t$ edges. For a fixed $t$, we define $\Delta: U \rightarrow Z_{3 t} \times Z_{2}$ on the vertex set $U=V \cup W$ as follows:

$$
\begin{aligned}
& \Delta\left(v_{j}\right)= \begin{cases}(j t, 0), & \text { for } 0 \leq j<t, \\
\left(t^{2}+1,0\right), & \text { for } j=t,\end{cases} \\
& \Delta\left(w_{j}\right)= \begin{cases}(j(t-1), 1), & \text { for } 0 \leq j<t, \\
\left(t^{2}+1,1\right), & \text { for } j=t,\end{cases}
\end{aligned}
$$

where the integers in the first components are considered modulo 3t. We now compute the difference list $\Delta(E)$ of $\delta$ defined as in (1). Hence each element of $Z_{3 t}$ appears in $\Delta(E)$ and the difference condition holds. Figure 3 illustrates the definition for the case $t=1$.

Obviously, the wrapped-condition (7) relative to $X=\left\{v_{0}, w_{0}\right\}$ and $Y=\left\{v_{t}, w_{t}\right\}$ holds as well and the translation parameter $\kappa=t^{2}+1$ is coprime to $3 t$ for any $t$. Therefore, $\Delta$ defines the desired wrapped $\Delta$-labelling of $H(1 ; t)$.

Theorem 5. ([9]) Let $t$ be a positive integer. For all $t$ there is a $(d, f)$-cluttered ordering of the complete bipartite graph $K_{3 t, 3 t}$ with $d=3$ and $f=4$.

Theorem 6. ([9]) Let $t$ be a positive integer. For all $t$ there is a $(d$, $f$ )-cluttered ordering of the complete bipartite graph $K_{3 t, 3 t}$ with $d=3 s+r$ and $f=2(s+1)+r, s>0, r=0,1,2$.

\subsection{A Sequence for $H(2 ; t)$}

We define a wrapped $\Delta$-labelling of $H(2 ; t)$ for any positive integer $t . H(2 ; t)=(U, E)$ has $4(t+1)$ vertices and $10 t$ edges. For a fixed $t$, a labelling $\Delta$ is a map $\Delta: U \rightarrow Z_{10 t} \times Z_{2}$ on the vertex set $U=V \cup W$. We specify the second component of $\Delta$ on the vertices $V=\left(v_{0}, v_{1}, \cdots, v_{2 t+1}\right)$ sequentially by the following list of $2 t$ +2 numbers:

$$
c_{0}, c_{0}+a, c_{1}, c_{1}+a, \cdots, c_{j}, c_{j}+a, \cdots, c_{t-1}, c_{t-1}+a, c_{0}+\kappa, c_{0}+a+\kappa,
$$

and, on the vertices $W=\left(w_{0}, w_{1}, \cdots, w_{2 t+1}\right)$ by, similarly,

$$
d_{0}, d_{0}+b, d_{1}, d_{1}+b, \cdots, d_{j}, d_{j}+b, \cdots, d_{t-1}, d_{t-1}+b, d_{0}+\kappa, d_{0}+b+\kappa,
$$

where we set

$$
\begin{aligned}
& a=6 t-1, \quad c_{j}=2 j t, \quad j=0,1, \cdots, t-1, \\
& b=6 t-2, \quad d_{j}=2 j(t-1), \quad j=0,1, \cdots, t-1, \\
& \kappa=2 t^{2}+1 .
\end{aligned}
$$

All integers are considered modulo 10t. Note that $|E|=10 t$ and $\kappa=2 t^{2}+1$ are coprime for all $t$ and that the wrapped-condition (7) is obviously fulfilled. Thus, $\Delta$ defines a wrapped $\Delta$-labelling.

Theorem 7. ([9]) Let $t$ be a positive integer. For all $t$ there is a $(d$, $f)$-cluttered ordering of the complete bipartite graph $K_{10 t, 10 t}$ with $d=10$ and $f=8$.

Theorem 8. ([9]) Let $t$ be a positive integer. For all $t$ there is a $(d, f)$-cluttered ordering of the complete bipartite graph $K_{10 t, 10 t}$ with $d=10 s+r$ and $f=4(s+1)+\min (r, 4), s>0, r=0,1, \cdots, 9$.

\subsection{A Sequence for $H(h ; 1)$}

We define in this section a wrapped $\Delta$-labelling for $H(h ; 1)$ for any positive integer $h . H(h ; 1)=(U, E)$ has $4 h$ vertices and $h(2 h+1)$ edges. We define the $\Delta$-labelling $\Delta: U \rightarrow Z_{h(2 h+1)} \times Z_{2}$ on the vertex set $U=V \cup W$ by specifying the first component of $\Delta$ on the vertices $V=\left(v_{0}, v_{1}, \cdots, v_{2 h-1}\right)$ sequentially by the following list of $2 h$ numbers:

$$
a_{0}, a_{1}, \cdots, a_{h-1}, a_{0}+\kappa, a_{1}+\kappa, \cdots, a_{h-1}+\kappa,
$$

and on the vertices $W=\left(w_{0}, w_{1}, \cdots, w_{2 h-1}\right)$ by, similarly, 


$$
b_{0}, b_{1}, \cdots, b_{h-1}, b_{0}+\kappa, b_{1}+\kappa, \cdots, b_{h-1}+\kappa,
$$

where we set

$$
\begin{array}{ll}
a_{0}=0, & a_{i}=2 i-(2 h+1), \quad i=1,2, \cdots, h-1, \\
b_{0}=0, & b_{j}=-j(2 h+1)-1, \quad j=1,2, \cdots, h-1, \\
\kappa=-1 . &
\end{array}
$$

All integers are considered modulo $h(2 h+1)$. Obviously, $|E|=h(2 h+1)$ and $\kappa$ are coprime for any positive integer $h$ and the wrapped-condition (7) is fulfilled. Figure 7 illustrates the definition for the case $h=3$. All numbers in $Z_{h(2 h+1)}$ appear exactly once as difference of $\boldsymbol{\Delta}$ which hence defines a wrapped $\boldsymbol{\Delta}$-labelling.

Theorem 9. ([9]) Let $h$ be a positive integer. For all $h$ there is $a(d, f)$-cluttered ordering of the complete bipartite graph $K_{h(2 h+1), h(2 h+1)}$ with $d=h(2 h+1)$ and $f=4 h$.

\section{Our Result: A Sequence of a Wrapped $\Delta$-Labelling for $H(3 ; t)$}

In this section, we define a wrapped $\Delta$-labelling of $H(3 ; t)$ for any positive integer $t . H(3 ; t)=(U, E)$ has $6(t+1)$ vertices and 21t edges. For a fixed $t$, a labelling $\Delta$ is a map $\Delta: U \rightarrow Z_{21 t} \times Z_{2}$ on the vertex set $U=V \cup W$. We specify the second component of $\Delta$ on the vertices $V=\left(v_{0}, v_{1}, \cdots, v_{3 t+2}\right)$ sequentially by the following list of $3 t+3$ numbers:

$$
c_{0}, c_{0}+a, c_{0}+2 a, c_{1}, c_{1}+a, c_{1}+2 a, \cdots, c_{j}, c_{j}+a, c_{j}+2 a, \cdots, c_{t-1}, c_{t-1}+a, c_{t-1}+2 a, c_{0}+\kappa, c_{0}+a+\kappa, c_{0}+2 a+\kappa,
$$

and, on the vertices $W=\left(w_{0}, w_{1}, \cdots, w_{3 t+2}\right)$ by, similarly,

$$
d_{0}, d_{0}+b, d_{0}+2 b, d_{1}, d_{1}+b, d_{1}+2 b, \cdots, d_{j}, d_{j}+b, d_{j}+2 b, \cdots, d_{t-1}, d_{t-1}+b, d_{t-1}+2 b, d_{0}+\kappa, d_{0}+b+\kappa, d_{0}+2 b+\kappa,
$$

where we set

$$
\begin{aligned}
& a=15 t-1, \quad c_{j}=3 j t, \quad j=0,1, \cdots, t-1, \\
& b=15 t-2, \quad d_{j}=3 j(t-1), \quad j=0,1, \cdots, t-1, \\
& \kappa=3 t^{2}+1 .
\end{aligned}
$$

All integers are considered modulo 21t. Note that $|E|=21 t$ and $\kappa=3 t^{2}+1$ are coprime for all positive integer $t$ and that the wrapped-condition (7) is obviously fulfilled. Figure 8 illustrates the definition for the case $t=1$.

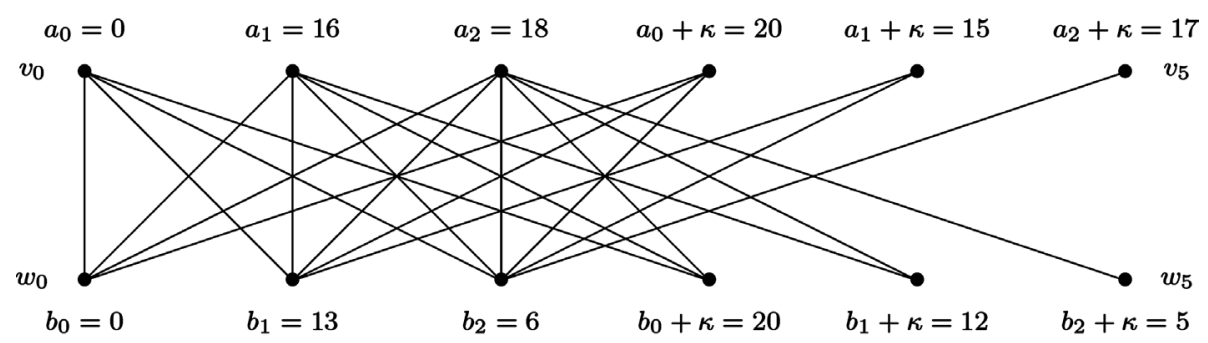

Figure 7. Some wrapped $\Delta$-labelling of $H(3 ; 1),|E|=21,|V|=12, \kappa=-1$.

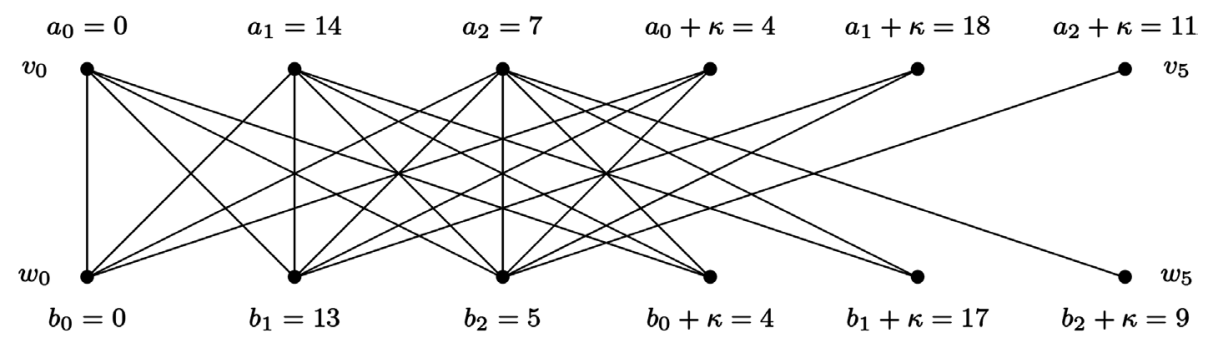

Figure 8. Some wrapped $\Delta$-labelling of $H(3 ; 1),|E|=21,|V|=12, \kappa=4$. 
We now compute the differences of $\Delta$ using the notation from (1):

$$
\begin{aligned}
\Delta\left(E_{0}^{\prime}\right)= & \left(c_{0}-d_{0}, c_{0}-d_{0}+(a-b), c_{0}-d_{0}+(2 a-2 b), c_{0}-d_{0}+a, c_{0}-d_{0}+2 a, c_{0}-d_{0}-b, c_{0}-d_{0}-2 b,\right. \\
& \left.c_{0}-d_{0}+(2 a-b), c_{0}-d_{0}+(a-2 b)\right) \\
= & (0,1,2,15 t-1,9 t-2,6 t+2,12 t+4,15 t, 6 t+3), \\
\Delta\left(E_{j}^{\prime}\right)= & \left(c_{j}-d_{j}, c_{j}-d_{j}+(a-b), c_{j}-d_{j}+(2 a-2 b), c_{j}-d_{j}+a, c_{j}-d_{j}+2 a, c_{j}-d_{j}-b,\right. \\
& \left.\quad c_{j}-d_{j}-2 b, c_{j}-d_{j}+(2 a-b), c_{j}-d_{j}+(a-2 b)\right) \\
= & (3 j, 3 j+1,3 j+2,3 j+15 t-1,3 j+9 t-2,3 j+6 t+2,3 j+12 t+4,3 j+15 t, 3 j+6 t+3) \\
& \quad \text { for } \quad j=1,2, \cdots, t-1, \\
\Delta\left(E_{j-1}^{\prime \prime}\right)= & \left(c_{j-1}-d_{j}, c_{j-1}-d_{j}+a, c_{j-1}-d_{j}+2 a, c_{j-1}-d_{j}+(a-b), c_{j-1}-d_{j}+(2 a-b), c_{j-1}-d_{j}+(2 a-2 b)\right) \\
= & (3 j+18 t, 3 j+12 t-1,3 j+6 t-2,3 j+18 t+1,3 j+12 t, 3 j+18 t+2) \\
\quad & \quad \text { or } j=1,2, \cdots, t-1, \\
\Delta\left(E_{j-1}^{\prime \prime \prime}\right)= & \left(c_{j}-d_{j-1}, c_{j}-d_{j-1}-b, c_{j}-d_{j-1}-2 b, c_{j}-d_{j-1}+(a-b), c_{j}-d_{j-1}+(a-2 b), c_{j}-d_{j-1}+(2 a-2 b)\right) \\
= & (3 j+3 t-3,3 j+9 t-1,3 j+15 t+1,3 j+3 t-2,3 j+9 t, 3 j+3 t-1) \\
\quad & \quad \text { for } \quad j=1,2, \cdots, t-1, \\
\Delta\left(E_{t-1}^{\prime \prime}\right)= & \left(c_{t-1}-d_{0}-\kappa, c_{t-1}-d_{0}-\kappa+a, c_{t-1}-d_{0}-\kappa+2 a, c_{t-1}-d_{0}-\kappa+(a-b), c_{t-1}-d_{0}-\kappa+(2 a-b),\right. \\
& \left.\quad c_{t-1}-d_{0}-\kappa+(2 a-2 b)\right) \\
= & (18 t-1,12 t-2,6 t-3,18 t, 12 t-1,18 t+1), \\
\Delta\left(E_{t-1}^{\prime \prime \prime}\right)= & \left(c_{0}+\kappa-d_{t-1}, c_{0}+\kappa-d_{t-1}-b, c_{0}+\kappa-d_{t-1}-2 b, c_{0}+\kappa-d_{t-1}+(a-b), c_{0}+\kappa-d_{t-1}+(a-2 b),\right. \\
\quad & \left.c_{0}+\kappa-d_{t-1}+(2 a-2 b)\right) \\
= & (6 t-2,12 t, 18 t+2,6 t-1,12 t+1,6 t) .
\end{aligned}
$$

We now compute the difference list $\Delta(E)$ :

$$
\begin{aligned}
& \Delta\left(E_{0}^{\prime}\right) \supset(0,1,2), \\
& \Delta\left(\bigcup_{j=1}^{t-1} E_{j}^{\prime}\right) \supset\{3 j, 3 j+1,3 j+2 \mid 1 \leq j \leq t-1\}=\{3,4,5, \cdots, 3 t-1\}, \\
& \Delta\left(\bigcup_{j=1}^{t-1} E_{j}^{\prime \prime \prime}\right) \supset\{3 j+3 t-3,3 j+3 t-2,3 j+3 t-1 \mid 1 \leq j \leq t-1\}=\{3 t, 3 t+1,3 t+2, \cdots, 6 t-4\}, \\
& \Delta\left(E_{t-1}^{\prime \prime}\right) \supset(6 t-3), \\
& \Delta\left(E_{t-1}^{\prime \prime}\right) \supset(6 t-2,6 t-1,6 t), \\
& \Delta\left(\bigcup_{j=1}^{t-1} E_{j-1}^{\prime \prime}\right) \supset\{3 j+6 t-2 \mid j=1\}=\{6 t+1\}, \\
& \Delta\left(E_{0}^{\prime}\right)-(1) \supset(6 t+2,6 t+3), \\
& \Delta\left(\bigcup_{j=1}^{t-1} E_{j-1}^{\prime \prime}\right)-(6) \supset\{3 j+6 t-2 \mid 2 \leq j \leq t-1\}=\{6 t+4,6 t+7,6 t+10, \cdots, 9 t-5\}, \\
& \Delta\left(\bigcup_{j=1}^{t-1} E_{j}^{\prime}\right)-(2) \supset\{3 j+6 t+2,3 j+6 t+3 \mid 1 \leq j \leq t-2\}=\{6 t+5,6 t+6,6 t+8,6 t+9, \cdots, 9 t-4,9 t-3\},
\end{aligned}
$$




$$
\begin{aligned}
& \left(\Delta\left(\bigcup_{j=1}^{t-1} E_{j-1}^{\prime \prime}\right)-(6)\right) \cup\left(\Delta\left(\bigcup_{j=1}^{t-1} E_{j}^{\prime}\right)-(2)\right) \\
& \supset(8-1) \cup(8-2) \\
& =\{3 j+6 t-2 \mid 2 \leq j \leq t-1\} \cup\{3 j+6 t+2,3 j+6 t+3 \mid 1 \leq j \leq t-2\} \\
& =(6 t+4,6 t+5,6 t+6,6 t+7,6 t+8,6 t+9,6 t+10, \cdots, 9 t-5,9 t-4,9 t-3), \\
& \Delta\left(E_{0}^{\prime}\right)-(1)-(7) \supset(9 t-2), \\
& \Delta\left(\bigcup_{j=1}^{t-1} E_{j}^{\prime}\right)-(2)-(8-2) \supset\{3 j+6 t+2,3 j+6 t+3 \mid j=t-1\}=(9 t-1,9 t), \\
& \Delta\left(\bigcup_{j=1}^{t-1} E_{j}^{\prime}\right)-(2)-(8-2)-(10) \supset\{3 j+9 t-2 \mid 1 \leq j \leq t-1\}=(9 t+1,9 t+4,9 t+7, \cdots, 12 t-5), \\
& \Delta\left(\bigcup_{j=1}^{t-1} E_{j-1}^{\prime \prime \prime}\right)-(3) \supset\{3 j+9 t-1,3 j+9 t \mid 1 \leq j \leq t-1\}=(9 t+2,9 t+3,9 t+5,9 t+6, \cdots, 12 t-4,12 t-3), \\
& \left(\Delta\left(\bigcup_{j=1}^{t-1} E_{j}^{\prime}\right)-(2)-(8-2)-(10)\right) \cup\left(\Delta\left(\bigcup_{j=1}^{t-1} E_{j-1}^{\prime \prime \prime}\right)-(3)\right) \\
& \supset(11-1) \cup(11-2) \\
& =\{3 j+9 t-2 \mid 1 \leq j \leq t-1\} \cup\{3 j+9 t-1,3 j+9 t \mid 1 \leq j \leq t-1\} \\
& =(9 t+1,9 t+2,9 t+3,9 t+4,9 t+5,9 t+6, \cdots, 12 t-5,12 t-4,12 t-3) \text {. } \\
& \Delta\left(E_{t-1}^{\prime \prime}\right)-(4) \supset(12 t-2,12 t-1) \text {, } \\
& \Delta\left(E_{t-1}^{\prime \prime \prime}\right)-(5) \supset(12 t, 12 t+1) \text {, } \\
& \Delta\left(\bigcup_{j=1}^{t-1} E_{j-1}^{\prime \prime}\right)-(6)-(8-1) \supset\{3 j+12 t-1,3 j+12 t \mid j=1\}=(12 t+2,12 t+3), \\
& \Delta\left(E_{0}^{\prime}\right)-(1)-(7)-(9) \supset(12 t+4), \\
& \Delta\left(\bigcup_{j=1}^{t-1} E_{j-1}^{\prime \prime}\right)-(6)-(8-1)-(14) \supset\{3 j+12 t-1,3 j+12 t \mid 2 \leq j \leq t-1\} \\
& =(12 t+5,12 t+6,12 t+8,12 t+9, \cdots, 15 t-4,15 t-3), \\
& \Delta\left(\bigcup_{j=1}^{t-1} E_{j}^{\prime}\right)-(2)-(8-2)-(10)-(11-1) \supset\{3 j+12 t+4 \mid 1 \leq j \leq t-2\}=(12 t+7,12 t+10, \cdots, 15 t-2), \\
& \left(\Delta\left(\bigcup_{j=1}^{t-1} E_{j-1}^{\prime \prime}\right)-(6)-(8-1)-(14)\right) \cup\left(\Delta\left(\bigcup_{j=1}^{t-1} E_{j}^{\prime}\right)-(2)-(8-2)-(10)-(11-1)\right) \supset(16-1) \cup(16-2) \\
& =\{3 j+12 t-1,3 j+12 t \mid 2 \leq j \leq t-1\} \cup\{3 j+12 t+4 \mid 1 \leq j \leq t-2\} \\
& =(12 t+5,12 t+6,12 t+7,12 t+8,12 t+9,12 t+10, \cdots, 15 t-4,15 t-3,15 t-2) \text {. } \\
& \Delta\left(E_{0}^{\prime}\right)-(1)-(7)-(9)-(15)=(15 t-1,15 t), \\
& \Delta\left(\bigcup_{j=1}^{t-1} E_{j}^{\prime}\right)-(2)-(8-2)-(10)-(11-1)-(16-2) \supset\{3 j+12 t+4 \mid j=t-1\}=(15 t+1), \\
& \Delta\left(\bigcup_{j=1}^{t-1} E_{j}^{\prime}\right)-(2)-(8-2)-(10)-(11-1)-(16-2)-(18) \\
& =\{3 j+15 t-1,3 j+15 t \mid 1 \leq j \leq t-1\} \\
& =(15 t+2,15 t+3,15 t+5,15 t+6, \cdots, 18 t-4,18 t-3) \text {, }
\end{aligned}
$$




$$
\begin{aligned}
& \Delta\left(\bigcup_{j=1}^{t-1} E_{j-1}^{\prime \prime \prime}\right)-(3)-(11-2)=\{3 j+15 t+1 \mid 1 \leq j \leq t-1\}=(15 t+4,15 t+7, \cdots, 18 t-2), \\
& \left(\Delta\left(\bigcup_{j=1}^{t-1} E_{j}^{\prime}\right)-(2)-(8-2)-(10)-(11-1)-(16-2)-(18)\right) \cup\left(\Delta\left(\bigcup_{j=1}^{t-1} E_{j-1}^{\prime \prime \prime}\right)-(3)-(11-2)\right) \\
& \quad=(19-1) \cup(19-2) \\
& \quad=\{3 j+15 t-1,3 j+15 t \mid 1 \leq j \leq t-1\} \cup\{3 j+15 t+1 \mid 1 \leq j \leq t-1\} \\
& \quad=(15 t+2,15 t+3,15 t+4,15 t+5,15 t+6,15 t+7, \cdots, 18 t-4,18 t-3,18 t-2), \\
& \Delta\left(E_{t-1}^{\prime \prime}\right)-(4)-(12)=(18 t-1,18 t, 18 t+1), \\
& \Delta\left(E_{t-1}^{\prime \prime \prime}\right)-(5)-(13)=(18 t+2), \quad=(18 t+3,18 t+4,18 t+5, \cdots, 21 t-1) . \\
& \Delta\left(\bigcup_{j=1}^{t-1} E_{j-1}^{\prime \prime}\right)-(6)-(8-1)-(14)-(16-1)=\{3 j+18 t, 3 j+18 t+1,3 j+18 t+2 \mid 1 \leq j \leq t-1\}
\end{aligned}
$$

From this one easily checks that the twenty-two lists cover all numbers in $Z_{21 t}$ exactly once. Thus, $\Delta$ defines a wrapped $\Delta$-labelling and by applying Proposition 4 we get the following result.

Theorem 10. Let $t$ be a positive integer. For all $t$ there is a $(d, f)$-cluttered ordering of the complete bipartite graph $K_{21 t, 21 t}$ with $d=21$ and $f=12$.

Using the same edge ordering of $K_{21 t, 21 t}$ one gets the following theorem by enlarging the window $d$.

Theorem 11. Let $t$ be a positive integer. For all $t$ there is a $(d$, $f)$-cluttered ordering of the complete bipartite graph $K_{21 t, 21 t}$ with $d=21 s+r$ and $f=6(s+1)+\min (r, 6), s>0, r=0,1, \cdots, 20$.

For example, we get a $(21,12)$-cluttered ordering of $K_{21 t, 21 t}$. For the graphs $K_{21 t, 21 t}$, this is a much better ordering than the $(21,16)$-cluttered ordering from Theorem 6.

\section{Conclusion}

In conclusion, we give a new sequence for construction of wrapped $\Delta$-labellings. Figure 7 and Figure 8 are the same as a graph, but they are different as a sequence. Cluttered orderings given by two sequences construct the different orderings for the complete bipartite graph $K_{21,21}$.

\section{Acknowledgements}

We thank the Editor and the referee for their comments.

\section{References}

[1] Hellerstein, L., Gibson, G., Karp, R., Katz, R. and Patterson, D. (1994) Coding Techniques for Handling Failures in Large Disk Arrays. Algorithmica, 12, 182-208. http://dx.doi.org/10.1007/BF01185210

[2] Chen, P., Lee, E., Gibson, G., Katz, R. and Ptterson, D. (1994) RAID: High-Performance, Reliable Secondary Storage. ACM Computing Surveys, 26, 145-185. http://dx.doi.org/10.1145/176979.176981

[3] Chee, Y., Colbourn, C. and Ling, A. (2000) Asymptotically Optimal Erasure-Resilient Codes for Large Disk Arrays. Discrete Applied Mathematics, 102, 3-36. http://dx.doi.org/10.1016/S0166-218X(99)00228-0

[4] Cohen, M. and Colbourn, C. (2000) Optimal and Pessimal Orderings of Steiner Triple Systems in Disk Arrays. Lecture Notes in Computer Science, 1776, 95-104. http://dx.doi.org/10.1007/10719839_10

[5] Cohen, M. and Colbourn, C. (2001) Ordering Disks for Double Erasure Codes. Proceedings of the Thirteenth Annual ACM Symposium on Parallel Algorithms and Architectures, 229-236.

[6] Cohen, M., Colbourn, C. and Froncek, D. (2001) Cluttered Orderings for the Complete Graph. Lecture Notes in Computer Science, 2108, 420-431. http://dx.doi.org/10.1007/3-540-44679-6_48

[7] Cohen, M. and Colbourn, C. (2004) Ladder Orderings of Pairs and RAID Performance. Discrete Applied Mathematics, 138, 35-46. http://dx.doi.org/10.1016/S0166-218X(03)00268-3

[8] Adachi, T. and Uehara, H. (2014) Construction of Wrapped $\rho$-Labellings for RAID. Journal of Mathematics and Sys- 
tem Science, 4, 750-754.

[9] Mueller, M., Adachi, T. and Jimbo, M. (2005) Cluttered Orderings for the Complete Bipartite Graph. Discrete Applied Mathematics, 152, 213-228. http://dx.doi.org/10.1016/j.dam.2005.06.005

[10] Adachi, T. (2007) Optimal Ordering of the Complete Tripartite Graph $K_{9,9,9}$. Proceedings of the Fourth International Conference on Nonlinear Analysis and Convex Analysis, 1-10.

[11] Bosák, J. (1990) Decompositions of Graphs. Kluwer Academic Publishers, Dordrecht. 
Scientific Research Publishing (SCIRP) is one of the largest Open Access journal publishers. It is currently publishing more than 200 open access, online, peer-reviewed journals covering a wide range of academic disciplines. SCIRP serves the worldwide academic communities and contributes to the progress and application of science with its publication.

Other selected journals from SCIRP are listed as below. Submit your manuscript to us via either submit@scirp.org or Online Submission Portal.
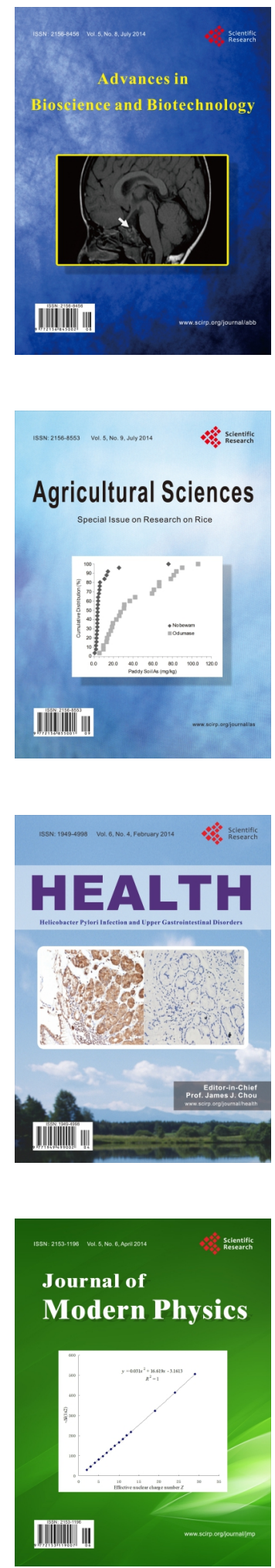
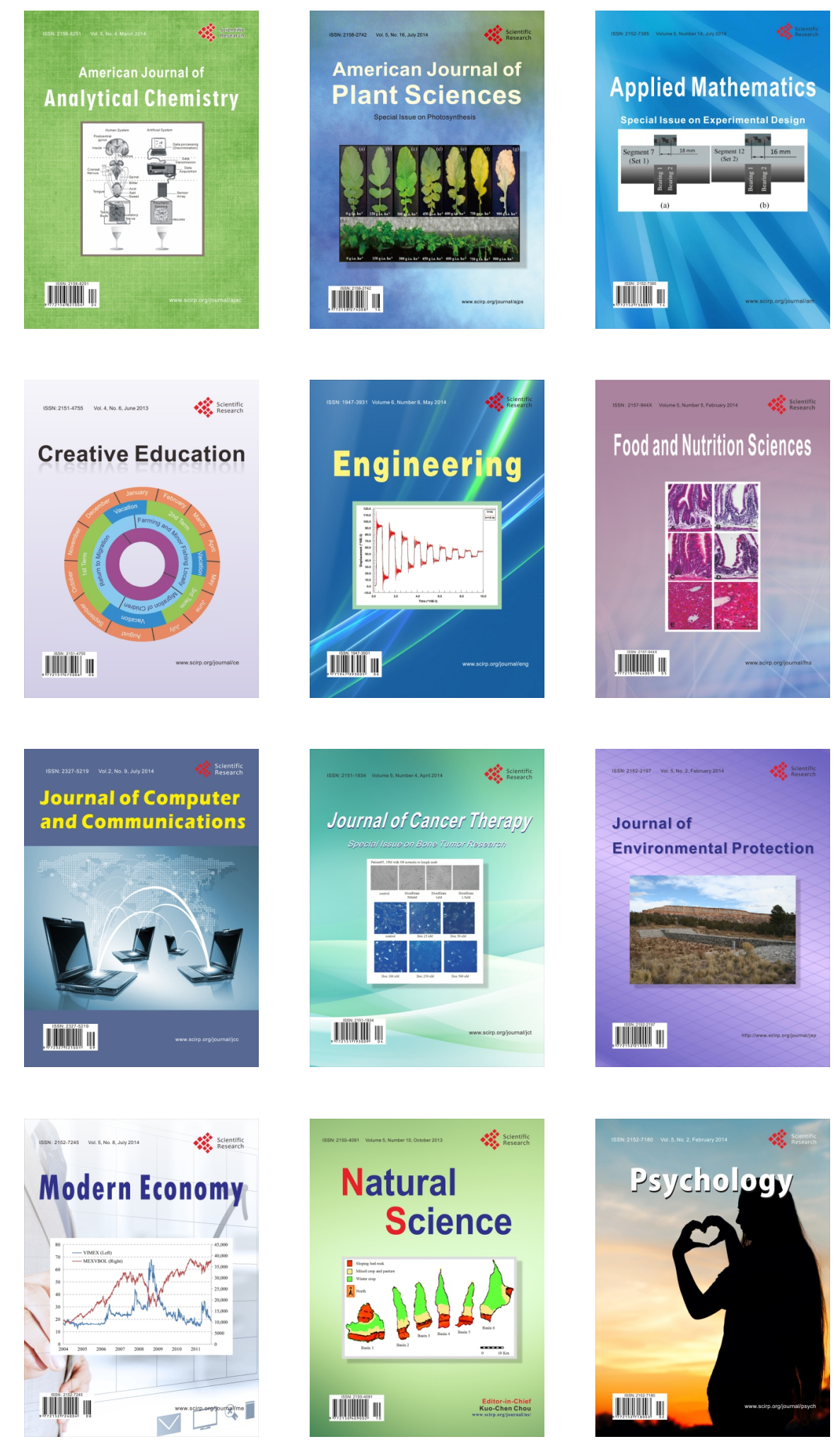